\title{
SOME EFFECTS OBSERVED IN THE MAGNETIC FLUID UNDER THE INFLUENCE OF QUASI-HOMOGENEOUS MAGNETIC FIELD
}

The effect of homogenous external magnetic fields on the created space structure of a nanoparticle distribution in a sample of magnetic fluid was studied. A grating was formed by the interference field of two crossed Ar-laser beams. The magnetic field was formed by using two electromagnets. The magnetic field oriented parallel to strips of grating does not significantly change the shape of nanoparticle grating. Perpendicular direction of magnetic field causes redistribution of the nanoparticles and in consequence of that the perpendicular nanoparticle "quasi-grating" arised.

\section{Introduction}

Magnetic fluids are colloidal suspensions of ferromagnetics nanoparticles dispersed in a nonmagnetic carrier liquid and the investigation of it is very interesting from several points of view. As the suspensions of magnetic nanoparticles, they exhibit liquid behaviour combined with the superparamagnetic properties. Consequently, they are useful in various applications, as the magnetic control of their flow can be realized by attainable values of the magnetic field strengths. Another application is spanning from sealants in rotary shaft to head dissipaters in loud speaker coils to carrier liquids for medical substances e.g. Nanoparticles of magnetic materials with the typical diameter of $10 \mathrm{~nm}$ are large enough to have extremely small particle mobility. This leads to a situation where the typical redistribution time constants are extremely large. On the other hand, the typical manifestation of these conditions is the behaviour of the nanoparticles in conditions of the created inhomogenities.

On top of it, fluids can be influenced by magnetic field. When additional energy is imported by a light beam and a spatial structure is thus created (grating for instance), it results in appearance of diffusion- and termodiffusion fluxes. The total nanoparticle flux will be given not only by its diffusion part but also by the properties of the termodiffusion mechanisms, mainly by the temperature behaviour of the Soret constant $[1,2]$ and their dependence on the magnetic field in the equation

$$
\begin{aligned}
& i=-D_{0}\left(1+D^{m}\right) \cdot \frac{\partial n}{\partial x}-D_{0}\left(1+D^{m}\right) . \\
& \cdot S_{T}\left(S_{T}^{0}+S_{T}^{m}\right) \cdot n \cdot \frac{\partial T}{\partial x}
\end{aligned}
$$

where the first term on the right hand side is the diffusion flux, the second is the thermo-diffusion flux of the particles. $D_{0}$ is the diffusion constant of particles, $S$ is the thermo-diffusion coefficient (the Soret constant), $n$ is the particle concentration, $T$ is the temperature and $D^{m}$ and $S_{T}^{m}$ take into consideration the influence of the magnetic field [3], [4].

Experimentally it can be verified that particles can accumulate (under specific conditions) at places of the lower temperature (for $S>0$ ), or at places where the temperature is higher (for $S<0$ ). The spatial redistribution of the nanoparticle concentration depends on sighn of parameter $S$. As far as the nanoparticle concentration explicitly affects the local optical properties of the ferrofluid, this can be used for the investigation of the processes mentioned above by means of optical methods.

\section{Experimental results}

In this article the study of nanoparticle kinetics in the magnetic liquid under the influence of the external magnetic field is presented. The study was carried out using a diagnostic laser beam $(\lambda=633 \mathrm{~nm})$ and its diffraction on the nanoparticle diffraction grating which was generated by two crossing Ar laser beams $(\lambda=$ $=488 \mathrm{~nm}$ ) through the interference field.

The relative position of the diffraction grating and magnetic induction vector $\vec{B}$ can be oriented according to Fig. 1 . The direction of the strings of the grating was always in the direction of $x$ axis, and the diagnostic $(\lambda=633 \mathrm{~nm})$ beam was always in the direction of $z$ axis. In this article the magnetic field influence in the axes $\boldsymbol{x}$ and $\boldsymbol{y}$ is presented only.

The experiments were carried out on a magnetic liquid sample based on kerosene with dispersed nanoparticles $\mathrm{Fe}_{2} \mathrm{O}_{3}$ of about $10 \mathrm{~nm}$ diameter. The thickness of the cell was about $65 \mu \mathrm{m}$.

\footnotetext{
* Julius Stelina, Ctibor Musil

Department of Physics, Faculty of Electrical Engineering, University of Zilina, Slovakia, E-mail: stelin@fel.uniza.sk
} 


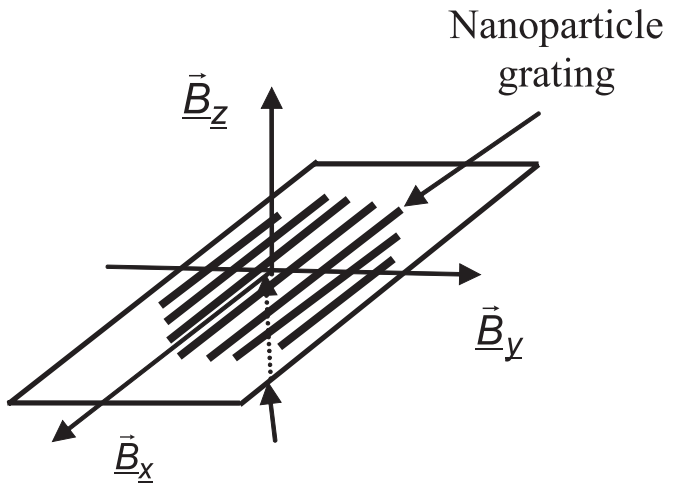

Fig. 1 Relative position of diagnostic laser beam, magnetic induction vector and nanoparticles grating

The basic scheme of the apparatus used for the study of nanoparticle kinetics in the dispersion fluids is in Fig. 2.

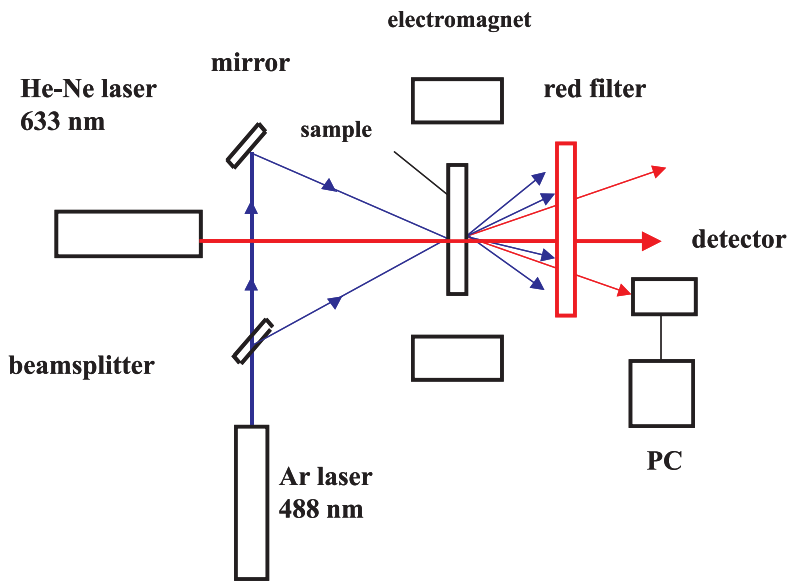

Fig. 2 The basic scheme of the apparatus

\subsection{The experimental results of the study for magnetic field $B_{x}$}

In this case the magnetic field is oriented in direction of the grating fringes ( $x$ direction) and lies in the plane of the created diffraction grating (see Fig. 1).

Fig. 3 shows a creation of the nanoparticle grating by the interference field of two crossed coherent laser beams with $\lambda=488 \mathrm{~nm}$ and its spontaneous decay. Fig. 4 presents the same case as Fig. 3 but the decay is under the influence of additional lighting beam $\lambda=488 \mathrm{~nm}$ and $6 \mathrm{~mW} / \mathrm{mm}^{2}$ power. We can see that the additional lighting beam stops or reduces the decay of the nanoparticle grating. Probably the thermo-diffusion (Soret) coefficient is negative in this phase of process.

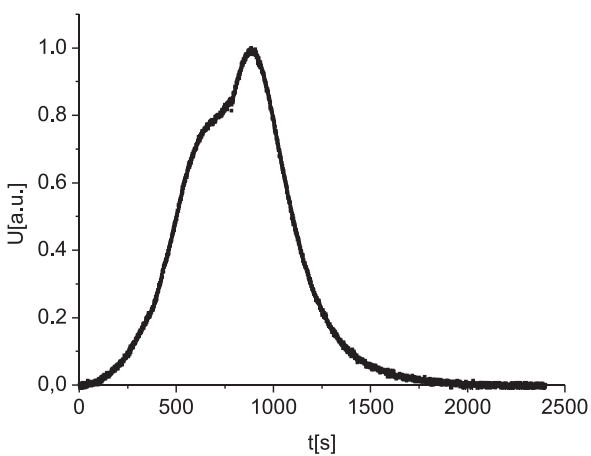

Fig. 3 The time dependence of the rise and decay of the nanoparticle grating without additional lighting

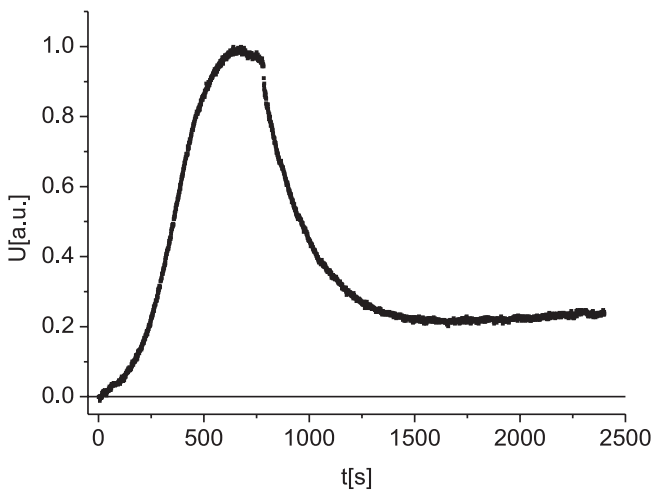

Fig. 4 The time dependence of the rise and decay of the nanoparticle grating with additional lighting of $6 \mathrm{~mW} / \mathrm{mm}^{2}$

The influence of the intensity of the magnetic field on the stability of the diffraction grating is presented by diffraction efficiency on the diffraction nanoparticle grating and is shown in Figs. 5.

Figs. $5 \mathrm{a}$ to $5 \mathrm{~d}$ show a decay of the nanoparticle grating under the influence of quasi-homogenous direct external magnetic field with values $8 \mathrm{mT}, 16 \mathrm{mT}, 24 \mathrm{mT}, 30 \mathrm{mT}$ and the additional lighting beam $\lambda=488 \mathrm{~nm}$ and $6 \mathrm{~mW} / \mathrm{mm}^{2}$ power was present as well. In the figures we can see that the presence of external magnetic

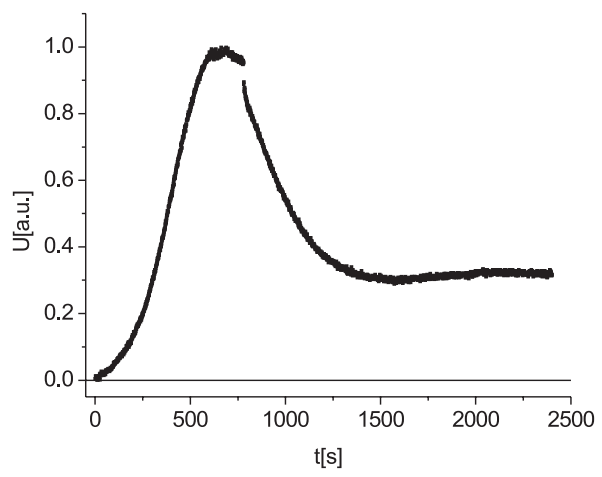

Fig. $5 a$ The decay of the grating at $B=8 m T$ 


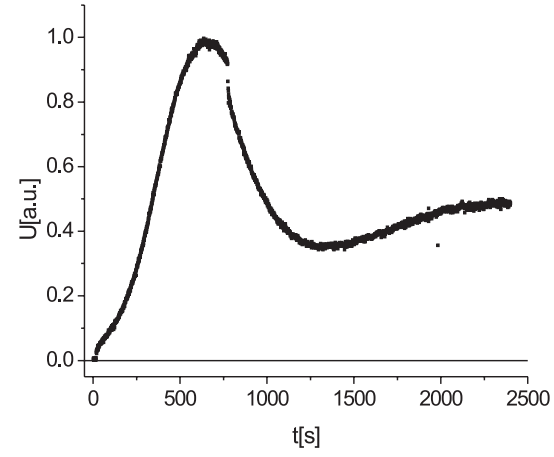

Fig. $5 b$ The decay of the grating at $B=16 \mathrm{mT}$

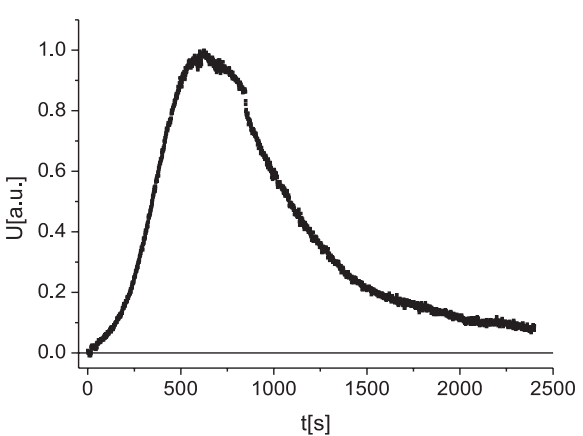

Fig. 5c The decay of the grating at $B=24 m T$

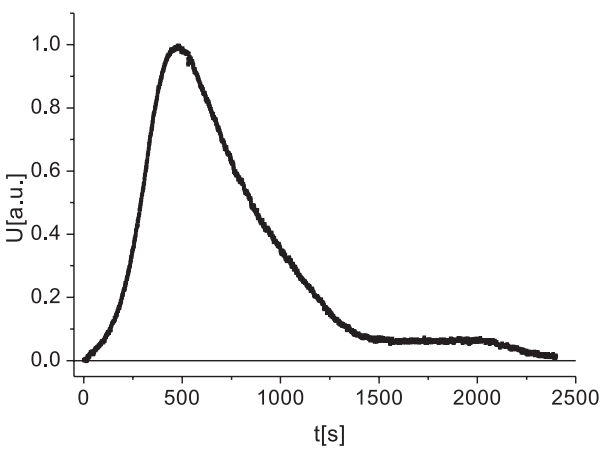

Fig. $5 d$ The decay of the grating at $B=30 \mathrm{mT}$

field at the magnetic induction higher than $16 \mathrm{mT}$ causes a quick decay of the nanoparticle grating and does not stabilise it.

The presence of the external magnetic field without additional lighting beam does not stabilise the nanoparticle grating at all.

\section{Conclusions}

1. As was discovered in the past [6], the additional lighting with a suitable wavelength, i.e. such wavelength that is absorbed in the sample and that induces the rise of a suitable temperature gradient between the strips of the grating, can stabilize the grating or eventually make it of a better quality.

2. But if at the same time a magnetic field is applied in the direction of the strips of the grating and in their plane, a better quality of the grating is observed at first. When the value of the magnetic field is above $16 \mathrm{mT}$, the stabilizing effect of the additional lighting is removed (Fig. 5d).

\subsection{The experimental results of the study for magnetic field $B_{y}$}

In this case the nanoparticle grating is oriented again in the $x$ direction and external magnetic field is oriented perpendicularly to the grating strips, i.e. in the y direction and lies in the plane of the created diffraction grating (see Fig. 1).

In Figs. 6 and 7 the created nanoparticle grating and diffraction on it are shown.

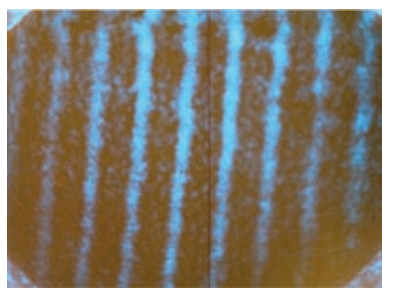

Fig. 6 Original nanoparticle grating

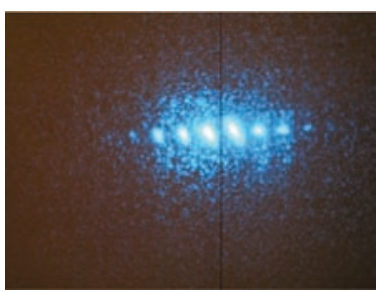

Fig. 7 Diffraction on the original grating
Fig. 8 shows the state of the nano-particle grating cca $5 \mathrm{~s}$ after switching on the perpendicular external magnetic field with $B=$ $=10 \mathrm{mT}$ intensity and Fig. 9 shows the diffraction on it. These figures illustrate a decay of the original grating. The decay of original grating is accompanied with the formation new one in perpendicular direction. This new grating is not exactly regular (therefore we call it "quasy-grating"). The diffraction on it is shown in Fig. 10.

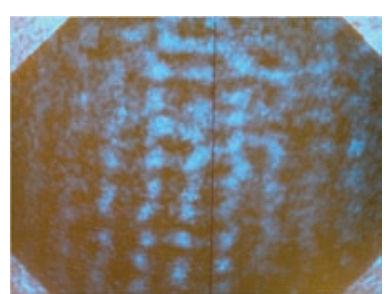

Fig. 8 The grating after switch on magnetic field.

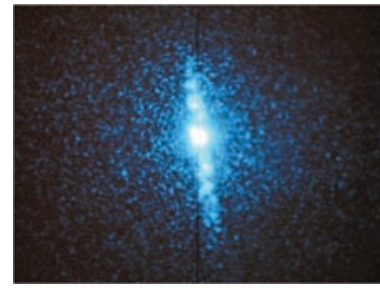

Fig. 10 Diffraction on the perpendicular new quasi-grating after some time.

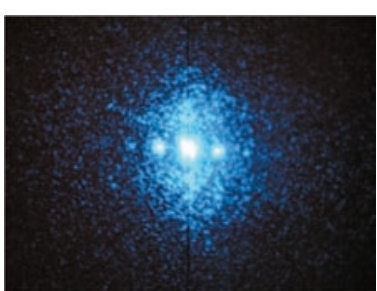

Fig. 9 The diffraction on the knew quasi-grating at the moment switch on of the magnetic field. 
The visage of the new "quasy-grating" $20 \mathrm{~s}$ and $1200 \mathrm{~s}$ after the external magnetic field was switched on can be seen in Figs. 11 and 12.

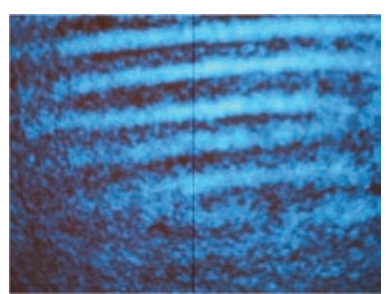

Fig. 11 The perpendicular

nanoparticle quasi- grating after $20 \mathrm{~s}$

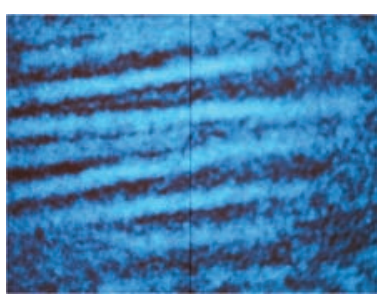

Fig. 12 The perpendicular nanoparticle quasi-grating after $1200 \mathrm{~s}$
The creation of the new "quasy-grating" in the time record of the diffraction efficiency can be seen in Fig. 13.

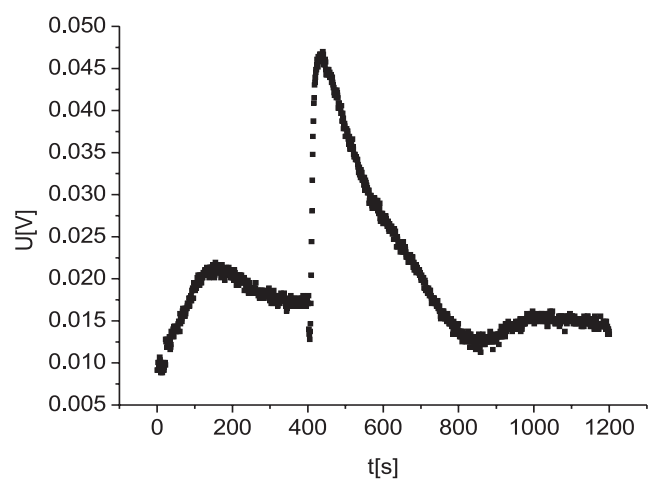

Fig. 13 The time dependence of the generation and decay of the first diffraction maximum which represent the arise of the perpendicular quasi-grating

The regular nanoparticle grating was generated through the interference field for the duration of $400 \mathrm{~s}$. The detector was placed into an expected position of the first diffraction maximum of the arising "quasi-grating". In the moment of $400 \mathrm{~s}$ (see Fig.13) one of the two interfering beams was switched off and the external magnetic field $10 \mathrm{mT}$ was switched on in the same time and

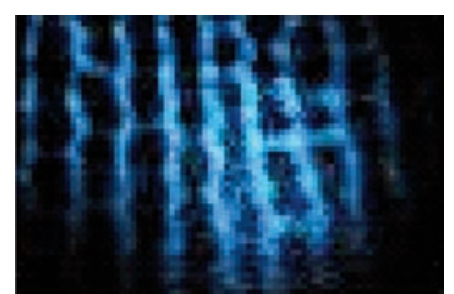

Fig. 14 The structural shape of the nanoparticle grating strings after $1080 \mathrm{~s}$ from the beginning of the additional lighting

the new "quasi-grating" arises very quickly (cca in $15 \mathrm{~s}$ ). In consequence of the used external magnetic field, which compensate influence of the additional lighting beam, the "quasi- grating" quick decay.

One argument for forming the perpendicular (in y direction oriented) "quasi-grating" is due to the cluster structure of strings of the original nanoparticle grating (illustrate Fig. 14). The influence of the external magnetic field probably causes transversal connection of the clusters in the strings. Because the cluster structure is not correctly periodical neither the forming transversal "quasi-grating" is not correctly periodical.

\section{Conclusions}

1. The nanoparticle grating was created in the sample of magnetic fluid.

2. To the created grating the transversal magnetic field $B=10 \mathrm{mT}$ and additional lighting $P_{L}=5 \mathrm{~mW} / \mathrm{mm}^{2}$ was applied.

3. Relatively quickly (15 s) the quasi-grating transversal to the original nanoparticle grating was generated and it was observed directly on the ground glass or through the diffraction on it.

4. Probably the quasi-grating is created due to the cluster structure of the nanoparticle grating (Fig. 14) [5] and the influence of the transversal magnetic field.

\section{Acknowledgements}

This work has been supported by Slovak Grant Agency VEGA under project No. 2/0077/09

\section{References}

[1] MEZUliS, A., BLUMS, E., BOURDON, A., DEMOUCHY, G.: Thermodiffusion- Induced Optical Index Grating in Ferrocolloids: Determination of Transport Coefficients, Proc. of Fourth International PAMIR conf. on MHDDTM, Presquile de Giens, France, 2000, 781

[2] KITA, R., KIRCHER, G., WIEGAND, S.: Thermally Induced Sign Change of Soret Coefficient for Dilute and Semidilute Solution of Poly(N-isopropylacrylamide) in Ethanol, J. Che. Phys. 121(18), 2004, p. 9140

[3] VOLKER, T., ODENBACH, S.: Physics of Fluids, Vol. 15, 8/2003

[4] BLUMS, E., ODENBACH, S., MEZULIS, A., MAIOROV, M.: Physics of Fluids, Vol. 10, 2155, 1998

[5] STELINA, J., MUSIL, C., BRACINIK, J., KOPCANSKY, P., TIMKO, M., TOMCO, L.: Proc. of $7^{\text {th }}$ International Conference on Applied Physics of Condensed Matter, 2006, p. 256.

[6] MUSIL, C., STELINA, J., KOPCANSKY, P., TIMKO, M.: The Influence of an External Paralel Magnetic Field on a Nanoparicle Grating Created in a Magnetic Fluid, $17^{\text {th }}$ Conference of Slovak Physicists, 2009, Bratislava, p. 109 